\title{
ASYMMETRICAL CHIRAL GAUGING TO INCREASE THE COEFFICIENT OF PERFORMANCE OF MAGNETIC MOTORS
}

\section{CALIBRE QUIRAL PARA AUMENTAR EL COEFICIENTE DE RENDIMIENTO DE MOTORES MAGNÉTICOS}

\author{
H. Torres-Silva ${ }^{1}$ \\ Recibido el 5 de septiembre de 2007, aceptado el 29 de noviembre de 2007 \\ Received: September 5, $2007 \quad$ Accepted: November 29, 2007
}

\begin{abstract}
RESUMEN
Este trabajo introduce un recalibre físico quiral asimétrico usado para aumentar el coeficiente de rendimiento de un motor eléctrico. Se presenta una revisión de la teoría de calibres y se examina el descarte de la condición de Lorentz para obtener el recalibrado quiral. Se introduce el coeficiente de rendimiento y se analiza un motor magnético bajo el enfoque quiral que permite un proceso Beltrami.
\end{abstract}

Palabras clave: Calibre quiral, motor magnético, Lorentz.

\begin{abstract}
This paper introduces a physical chiral asymmetrical regauging to increase the coefficient of performance of an electric motor. A review of gauge theory and a consideration of the disposal of the Lorentz condition to achieve the chiral regauging are presented. The coefficient of performance terminology is introduced. A magnetic motor is discussed under a chiral approach which gives a Beltrami process.
\end{abstract}

Keywords: Chiral gauge, magnetic motor, Lorentz.

\section{INTRODUCTION}

In this paper we investigate a process referenced in recent permanent magnet (PM) motor patents [1]. These specially designed PM motors claim to capture and use environmental energy as an additional energy input. The technique that allows this energy transfer to occur is called asymmetrical regauging (ASR). The physics behind the ASR process will be examined by reviewing gauge theory, the Lorentz gauge, and the effect of discarding the Lorentz gauge to include the vacuum chiral current density. The term coefficient of performance (COP) is introduced to adequately describe the energy transfer of these motors.

\section{REVIEW OF THE LORENTZ GAUGE}

To understand how environmental energy may be utilized in a motor, to theoretically gain a $\mathrm{COP}>1$, a review of the Lorentz gauge is first presented. The equations used in standard practice to design motors are derived from
Maxwell's equations. It has been accepted practice, to apply the Lorentz gauge to these equations to make them simpler. In abbreviated steps, we start with Maxwell's equations [14].

All the information in Maxwell's four equations can be reduced to the following equation:

$$
\left(\nabla^{2} \mathbf{A}-\mu_{0} \varepsilon_{0} \frac{\partial^{2} \mathbf{A}}{\partial t^{2}}\right)-\nabla\left(\nabla \bullet \mathbf{A}+\mu_{0} \varepsilon_{0} \frac{\partial V}{\partial t}\right)=-\mu_{0} \varepsilon_{0} \mathbf{J}
$$

The Lorentz gauge is then applied to reduce the complexity of these two equations. Mathematically, applying any gauge, is represented by $(2,3)$ where gamma is an arbitrary, differentiable scalar function called the gauge function [5].

$$
\begin{aligned}
& V(t, x) \mapsto V^{\prime}(t, x)=V(t, x)-\frac{\partial \Gamma(t, x)}{\partial t} \\
& \mathbf{A}(t, x) \mapsto \mathbf{A}^{\prime}(t, x)=\mathbf{A}(t, x)+\nabla \Gamma(t, x)
\end{aligned}
$$

1 Instituto de Alta Investigación. Universidad de Tarapacá. Antofagasta No 1520. Arica, Chile. E-mail: htorres@uta.cl 
To specifically apply the Lorentz gauge, the "Lorentz Condition" is imposed by choosing a set of potentials $(A, V)$ such that

$$
\nabla \bullet \mathbf{A}=\mu_{0} \varepsilon_{0} \frac{\partial V}{\partial t}
$$

Equations (4) are the ones on which all the equations for motor design are currently based. Since the magnetic vector field and the voltage scalar field are both changed at the same time, this can be referred to as symmetrical gauging, so

$$
\begin{gathered}
\nabla^{2} \mathbf{A}-\mu_{0} \varepsilon_{0} \frac{\partial^{2} \mathbf{A}}{\partial t^{2}}=-\mu_{0} \boldsymbol{J} \\
\nabla^{2} V-\mu_{0} \varepsilon_{0} \frac{\partial^{2} V}{\partial t^{2}}=-\frac{1}{\varepsilon_{0}} \rho
\end{gathered}
$$

Notice that (5) is (1) with the middle term, (7), eliminated.

$$
-\nabla(\nabla \bullet \mathbf{A})+\mu_{0} \varepsilon_{0} \frac{\partial V}{\partial t}
$$

\section{ASYMMETRICAL REGAUGING}

Invoking the Lorentz condition in classical electromagnetics discards the vacuum polarization component that exists in quantum electrodynamics [6] since

$$
\begin{gathered}
-\nabla(\nabla \bullet \mathbf{A})+\mu_{0} \varepsilon_{0} \frac{\partial V}{\partial t}=\mu_{0} \mathbf{j} \\
\text { and } \mathbf{J}_{A}=\sigma \mathbf{E}_{A}
\end{gathered}
$$

Asymmetrical regauging is the equivalent of discarding the Lorentz condition. Further ASR is any process that changes the potential energy of a system and also produces a net force in the process [6].

Understanding the vacuum and its polarization are essential steps to utilizing energy from the environment. According to T.D. Lee, he define the vacuum state as the lowest energy state of the system [7]. Hence, the vacuum is considered to be the worst case model of the environment. Maxwell's equations must be modified, in the vacuum, since $\rho$ and $\mathbf{J}$ vanish. Classically, this causes the Ampere-Maxwell law to be revised.

$$
\nabla \times \mathbf{B}=\mu_{0} \varepsilon_{0} \frac{\partial \mathbf{E}}{\partial t}
$$

In $[6,8]$, the authors show that if the vacuum current density factor is included, the above equation changes to

$$
\nabla \times \mathbf{H}=\mathbf{j}_{A}+\frac{\partial \mathbf{D}}{\partial t}
$$

where $\mathbf{D}=\varepsilon_{0} \mathbf{E}+\mathbf{P}_{A}$ and $\mathbf{B}=\mu_{0} \mathbf{H}+\mu_{0} \mathbf{M}$.

This leads to the result that

$$
\frac{\partial \mathbf{P}_{A}}{\partial t}=j_{A}=\frac{\partial(\varepsilon T \nabla \times \mathbf{E})}{\partial t} .
$$

Here we are considered for $\mathbf{D}, \mathbf{B}$ a chiral term so, [14]

$$
\vec{D}=\mathbf{D}=\varepsilon_{0} \mathbf{E}+\mathbf{P}_{A}=\varepsilon_{0} \mathbf{E}+\varepsilon T \nabla \times \mathbf{E}
$$

and

$$
\mathbf{B}=\mu_{0} \mathbf{H}+\mu_{0} \mathbf{M}=\mu_{0} \mathbf{H}+\mu_{0} T \nabla \times \mathbf{H} .
$$

Hence, $\mathrm{T}$ is the chiral factor which allows to extract vacuum energy, Thus discarding the Lorentz condition in classical electrodynamics leads to new equations that include the effect of the vacuum polarization.

\section{COEFFICIENT OF PERFORMANCE}

The energy transfer of electrical machinery is generally described using the term "efficiency". Efficiency is defined as the power output divided by the total power input from all sources. The underlying assumption when defining the energy of any system is that all the energy input is from an identifiable and measurable energy sources(s). In an ideal system the efficiency would be one. The equation for efficiency $(\eta)$ is normally stated [2] as

$$
\eta=\frac{P_{\text {Out }}}{P_{\text {In }}}[\text { Watts }] .
$$

Coefficient of performance is a broader energy transfer term that defines the measure of energy output divided by the operator's energy input. COP is used to describe any machinery that has additional energy input from the environment. For example, COP is commonly used to describe the energy exchange of heat pumps [3] or solar collectors. Unlike the term "efficiency", the COP can be greater than one. See figure 1 for the energy flow diagram. The following equation defines COP mathematically. 


$$
C O P \equiv \frac{P_{\text {Out }}}{P_{\text {In(Operator })}}[\text { Watts }]
$$

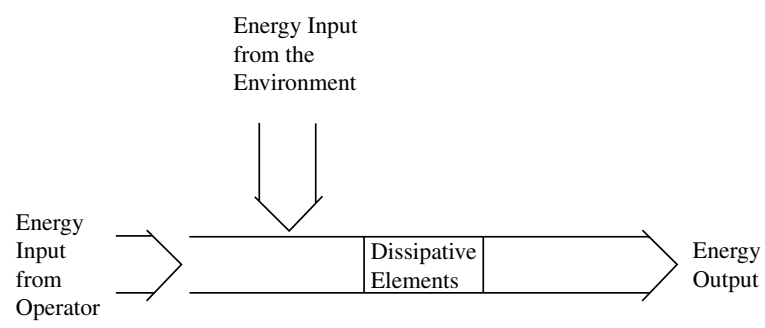

Figure 1. Energy flow for machines described by COP.

\section{CHIRAL MAGNETIC RESONANCE EFFECTS}

Besides electrical spacetime devices, self-running magnetic motors have been constructed in a repeatable and reproducible way ([4], see also figure 2, [1, 2]). The functioning of these devices cannot be explained by Maxwell-Heaviside electrodynamics but it can be explained with the chiral electrodynamics.

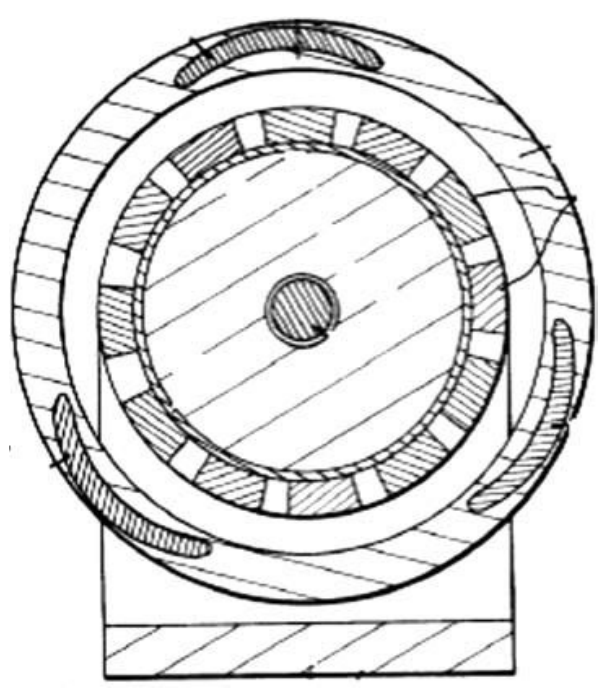

Figure 2. Johnson magnetic motor [1] Schematic representation of spacetime vector potential for a magnetic assembly: magnet stator including rotor magnets, flow with vortices (force field).

As was described in the preceding section of this edition, the Cartan torsion of spacetime introduces the spin connection as an additional quantity occurring in the laws of nature so that they take a generally covariant form. In particular this holds for the magnetic field. In Maxwell
Heaviside theory the magnetic field $\boldsymbol{B}$ is connected with its generating vector potential $\boldsymbol{A}$ by the relation

$$
\boldsymbol{B}=\nabla \times \boldsymbol{A}
$$

In TTS theory this law has to be replaced in the simplest case by [14-16].

$$
\mathbf{B}=\nabla \times(1+\boldsymbol{T} \nabla \times) \boldsymbol{A}
$$

where $\omega$ is the spin connection vector again. In the following we discern between the magnetic field of the assembly and the magnetic field of the surrounding spacetime itself, denoted by $\boldsymbol{B}_{s}$. The torque T acting on the magnetic dipolo moment $\mathrm{m}$ of the assembly due to the external field $\boldsymbol{B}_{s}$ is

$$
T=m \times B_{s}
$$

Under normal conditions there is no resulting torque because of $\boldsymbol{B}_{s}=0$. Spacetime is force free and does not bear a magnetic field. So there is no rotation of stationary magnets. The situation becomes different if it were possible to create a magnetic field from spacetime. In order to understand how this can be achieved we have first to look closer on the fields of the surrounding spacetime. In case of $\boldsymbol{B}_{s}=0$ it follows from Eq. (19) that

$$
\nabla \times \boldsymbol{A}=-\frac{1}{T} \boldsymbol{A}
$$

where $\boldsymbol{A}$ is the vector potential of the spacetime itself. In contrast at Maxwell-Heaviside theory, this is no gaugable quantity but is uniquely defined and has a physical meaning. In case of $\boldsymbol{A}$ consisting of plane waves, $\boldsymbol{\omega}$ takes a special form and Eq. (21) can be expressed as $\nabla \times \boldsymbol{A}=k_{B} \boldsymbol{A}$ with a wavelength $k_{B}=1 / T$, [14].

This equation is known as Beltrami equation in the literature [7]. It describes a flow with longitudinal vortices where streamlines have a helical form. In the case of chiral potential this means that there is no force field present, in accordance with our prior assumption.

Taking the curl at both sides of Eq. (21) gives

$$
\nabla \times(\nabla \times \boldsymbol{A})=\nabla \times\left(-\frac{1}{T} \boldsymbol{A}\right) \Rightarrow\left(\nabla^{2}+k_{B}^{2}\right) \boldsymbol{A}=0
$$

This is a Helmholtz equation for the spacetime surrounding the magnetic assembly. Because of the assumption of 
$\boldsymbol{B}_{s}=0$ there is no torque on the magnets, they remain at rest. Torque can be created by disturbing the Beltrami flow. For the Helmholtz equation this means that the balance to zero is no more fulfilled. Assuming a periodic imbalance leads to

$$
\left(\nabla^{2}+\frac{1}{T^{2}}\right) \boldsymbol{A}=\boldsymbol{R} \cos (\boldsymbol{k} \cdot \boldsymbol{r})
$$

with a vector $\mathrm{R}$ having units of inverse square meters, therefore it can be interpreted as a curvature. $\kappa$ is a wave vector and can be interpreted as the frequency of a driving force which the right hand side of the equation constitutes. If restricted to one coordinate $(\mathrm{x})$ the equation reads

$$
\left(\frac{\partial^{2}}{\partial x^{2}}+\frac{1}{T^{2}}\right) A_{\boldsymbol{x}}=R_{\boldsymbol{x}} \cos (k r)
$$

It can be seen that this is a differential equation for a resonance without damping $(\alpha=0)$. The resonant oscillation occurs in case $\mathrm{k}=1 / \mathrm{T}$ with $A_{x}$ going to infinity.

Because of violating the Beltrami condition, $\boldsymbol{A}$ creates a force field according to Eq. (24), which creates a torque being big enough to spin the magnetic assembly and to maintain the rotation. This is the mechanism how spacetime is able to do work via a resonance mechanism.

In total we have shown qualitatively how energy can be obtained from spacetime via magnetic assemblies. This could be the basis for development of an engineering for such devices.

It has already been shown in quantum electrodynamics that the vacuum behaves like a dielectric [9]. The vacuum sprouts positron-electron pairs as shown in the Feynman diagram.

It has been shown that by discarding the Lorentz gauge, the Ampere-Maxwell law equation evolves to include the current density of the vacuum. Also, the task remains to develop the equation and determine the process to apply it to magnetic motors. Future work is planned to study the magnetic motor to ascertain the exact mechanism involved that allows this motor to exchange energy with the vacuum.

\section{CONCLUSION}

It has been suggested in at least one recent patent that it is possible to make use of energy from the environment as an extra source in permanent magnet motors. This paper presents a new term "coefficient of performance' which may be used to more adequately describe the energy transfer of such an electromechanical system. This paper also shows the physics behind one possible explanation for this phenomenon.

The physics is explained by first considering how the Lorentz gauge is used to give us the design equations used today. The Lorentz gauge is then discarded to show how the current density of the vacuum may be included in the Maxwell-Ampere equation. They term asymmetrical regauging is introduced for this procedure. The particle physics explaining the vacuum polarity is introduced. A thorough investigation for practical application of this new equation is encouraged by suggestion that further study be applied to the "Wankel motor". Future work is planned to study the magnetic motor to ascertain the exact mechanism involved that allows this motor to exchange energy with the vacuum.

\section{REFERENCES}

[1] J. Bedini. "Device and Method of a Back EMF Permanent Electromagnetic Motor Generator". US: Bedini Technology, Inc. 2002.

[2] A. Trzynadlowski. "Introduction to Modern Power Electronics”. New York: John Wiley \& Sons. Inc. 1998.

[3] K. Annamalai and I.Puri, "Advanced Thermodynamics Engineering”. New York. CRC Press. 2002.

[4] D. Griffiths. "Introduction to Electrodynamics". New York. Prentice-Hall. 1999.

[5] B. Thide. "Electromagnetic Field Theory". Uppsala: Upsilon Books. 2004.

[6] P.K. Anastasovski. "Classical Electrodynamics without the Lorentz Condition: Extracting Energy from the Vacuum", Physica Scripta. Vol. 61, p. 513. 1999.

[7] T.D. Lee. "Particle Physics and Introduction to user Field Theory". New York: Harwood Academic Publishers. 1981. 
[8] B. Lehnert and S. Roy. "Extended Electromagnetic Theory”. Singapore: World Scientific. 1998.

[9] D. Griffiths. "Introduction to Elementary Particles". New York. John Wiley \& Sons, Inc. 1987.

[10] M.W. Evans and H. Eckardt. "Spin connection resonance in magnetic motors". Documento 74 en la serie sobre ECE. URLs: www.aias.us

[11] D. Reed. "Beltrami vector fields in electrodynamics - a reason for reexamining the structural foundations of classical field physics?" Modern Nonlinear Optics, Part 3. Second Edition. Advances in Chemical Physics. Vol. 119. Recopilado por Myron W. Evans. John Wiley \& Sons. 2001.

[12] G. Kasyanov. Phenomenon of electrical current rotation in nonlinear electric systems, Comment on the Violation of the law of charge conservation in the system, New Energy Technologies. Vol. 2 No 21, pp. 28-30. 2005.

[13] "Motor de Johnson de imanes permanentes". US Patent 4151431. 1979.

[14] H. Torres-Silva. "Electrodinámica quiral: eslabón para la unificación del electromagnetismo y la gravitación". Ingeniare. Rev. chil. ing. Vol. 16 $\mathrm{N}^{\mathrm{o}}$ 1, pp. 6-23. 2008.

[15] H. Torres-Silva. "Chiral field ideas for a theory of matter". Ingeniare. Rev. chil. ing. Vol. $16 \mathrm{~N}^{\mathrm{o}} 1$, pp. 36-42. 2008.

[16] H. Torres-Silva. "A metric for a chiral potential field”. Ingeniare. Rev. chil. ing. Vol. $16 \mathrm{~N}^{\circ} 1$, pp. 91-98. 2008. 